\title{
Support Vector Machine Based Route Classification and Description
}

\author{
Reshma M, Pristy Paul T, Surekha Mariam Varghese
}

\begin{abstract}
Traveling is very much important in one's life. Location-based services have developed a lot due to the development of communication technologies. It confines services that execute programs that use geographical data. Map services authorize travelers to look for the information surrounding them and organize an outing to his/her best-loved spot. Google Maps API is convenient for locating the shortest route information. However, the map system doesn't supply any illustration about air quality or congestion in a path. At times, a substitute route with less congestion can take you quickly to your spot than a shorter route. Numerous crucial health concerns for human beings are caused due to pollution. The motive is to develop a system that offers the textual explanation of routes utilizing the sub-routes information from Google map and BreezoMeter. The end-user can choose the starting and ending points of his/her travel and the route map showing various routes from source to destination is exhibited along with a small description of each route. The illustration of the routes is obtained depending on three factors such as air quality, congestion and distance gathered from BreezoMeter, Google map traffic API and distance matrix API respectively. Multicategory Support Vector Machine (SVM) is an organized and guided categorization technique and is used here to classify factors into various levels. Since the textual illustration of the route is accessible, the end-user can effortlessly understand the details about the route and they can choose a particular route.

Keywords: BreezoMeter, Google Maps, SVM, Traffic Congestion
\end{abstract}

\section{INTRODUCTION}

Traveling is a spectacular episode in everyone's life and gives each and every person wonderful experience. There is nothing very much like traveling, particularly seeing beautiful places, experiencing and enjoying different cultures or moving to the unique and loving place of everyone. Traveling has become an inescapable part of the life of everyone. People want to fly and explore new paths, regardless of their age and position in society. The motives for travel include fun, leisure, excursions, investigative travel and other factors such as

Revised Manuscript Received on April 25, 2020.

* Correspondence Author

Reshma M*, Computer Science and Engineering, Mar Athanasius College of Engineering, Kothamangalam, Ernakulam, India. Email: reshmamohandas96@gmail.com

Pristy Paul T, Computer Science and Engineering, Mar Athanasius College of Engineering, Kothamangalam, Ernakulam, India. Email: pristypault@gmail.com

Dr. Surekha Mariam Varghese, Computer Science and Engineering, Mar Athanasius College of Engineering, Kothamangalam, Ernakulam, India. Email: surekh.var@gmail.com

(C) The Authors. Published by Blue Eyes Intelligence Engineering and Sciences Publication (BEIESP). This is an open access article under the CC BY-NC-ND license (http://creativecommons.org/licenses/by-nc-nd/4.0/) getting healthcare or traveling pleasure. The excellent and impressive furtherance in the field of communication technologies has made potent growth in LBS. LBS provides various services to its users by utilizing geographical details. Maps related services helps the travelers to look for various routes to their destinations and as well as finding available facilities in each and every locations. Existing services automatically provide the best route to a particular destination by taking different parameters into consideration without providing any description.

Map support is the way maps are rendered network available. Within many applications, a map service makes graphics, functions and system attributes available. One common use of map service is viewing ArcGIS Online, Bing Maps or Google Maps business data on top of main map tiles. Map services are intended to work in various network and intranet situations. Interactive maps are created at the moment a person demands them. These are not as reliable as stored maps but can be suitable for highly focused intranet apps and applications involving real-time data display.

Multiple sensors are available at an air pollution monitoring station. BreezoMeter aims to improve the safety of billions of people around the world by providing real-time, location-based, actionable air quality info. A huge volume of proof of air pollution is being produced, and new techniques have been created to translate it into actionable information. These monitoring stations and many other networks collect data and calculate location-based air quality data in order to understand what the air they breathe means, irrespective of how far one is from a location. In addition to data from these proven monitoring stations, various information rates are obtained from a variety of sources, satellites, local weather, and traffic conditions. While millions of air-related data points exist, any system can hardly analyze them in a meaningful way. The consequence is a single number, distributed around the globe, between 0 and 100, which is a score for how pure the air you are currently breathing is.

Multi-class or multinomial classification is the problem of categorizing instances into one of three or more classes. Multi-class SVM aims to help SVM to assign labels to instances where the labels are taken from a specified set of different components. This can be applied by growing the single multi-class problem by one-versus-all into several binary classification problems.

The project aims to build a system that uses Google Maps and BreezoMeter information to give a textual definition of a road. The system helps a user who is unfamiliar with the path to better understand the path and allows users to select the best path to travel according to their needs.

The source and target may be defined by the user.

Published By:

Blue Eyes Intelligence Engineering DOI: $10.35940 /$ ijeat.C6286.049420

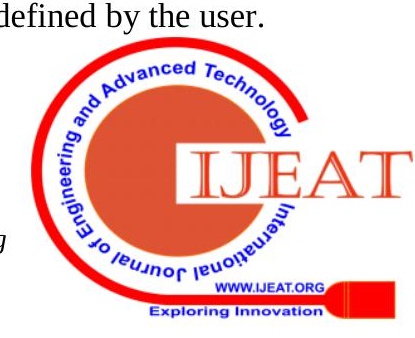


The overview of the routes is shown when the user enters the source and destination into the application. Several routes from one source to a destination are possible. All such routes are shown and by reaching any waypoint along the road, the user may pick one of them and the overview of the chosen route is shown, which includes details of all the subroutes along the path and the associated air quality and congestion.

\section{RELATED WORKS}

\section{A. Traffic Congestion}

An inductive loop is a contact using electromagnetism technology and to detect the device using a moving magnet to cause an electrical current in a nearby wire [1]. Inductive loops are used for transmission and for the identification of metal objects in metal detectors or vehicle presence indicators. Inductive loops are mounted in a roadbed to distinguish vehicles traveling over the loop, while more sophisticated sensors measure the vehicles' speed, length, and weight and distance. Another common technology is the use of cameras and image processing for vehicle counts. At a high altitude, one or more cameras are mounted in an airplane so that all points on the road are visible [2]. The camera captures optical data from both the visible and infrared spectra. The video-based system is an extension of this approach. The video recorded from a busy road is being closely analyzed and studied to identify the congestion. Cam-based frameworks are capable of differentiating numbers and arranging vehicles [3]. Such frameworks use feature image processors to identify vehicles and their criteria for streaming operations by analyzing the symbolism given by cam recorders. Pictures given by cams are digitized and calculations transforming the image are connected to them after that arrangement. While cam-based frameworks are more reliable than circle-based frameworks, they do have a few drawbacks. Magnetic sensors are capable of detecting a vehicle [4]. A vehicle is marked with this approach using variations in the Earth's magnetic field induced by moving vehicles. Wireless magnetic sensor networks provide an enticing, minimal-cost substitute to inductive loops, video, and radar for traffic monitoring, intersection and parking lots. The network consists of sensor nodes that are attached to the pavement in which vehicles are to be located. The sensor nodes transmit their data via radio to the roadside access points. AP forwards sensor data through GPRS or the roadside controller to the Traffic Management Centre. Since such networks can be implemented in a very short time, they can also be used and reused to monitor temporary traffic. Probe taxis are used to monitor traffic information in real-time and provide meaningful information about the region's traffic conditions such as travel information, best routes, etc. In addition to other information, GPS systems were mounted in probe taxis to collect spatial and temporal information every 3 to 5 seconds [5]. This provides real-time traffic information by measuring these probe taxis' spatial and temporal information, the spatial information includes the taxis' latitude and longitude coordinates, while the temporal information includes the UNIX epoch time. Simultaneously, other information such as system ID, speed, distance, taximeter, etc. is gathered from the probe taxis.

\section{B. Air Quality}

Ozone, PM2.5, nitrogen dioxide, sulfur dioxide, and carbon monoxide are significant contaminants to the air. Semiconductor sensors are used to detect automotive pollutant levels [6]. There are primarily three blocks: a smoke detector that detects contaminants, a microcontroller that compares pollutant levels to permissible levels. When that amount reaches a certain limit, the microcontroller sends the fuel injector a signal. The third block is a fuel injector that blocks the engine's fuel supply for a short period of time.

\section{Support Vector Machine}

A Support Vector Machine (SVM) is a conventionally defined selective classifier with a separating hyperplane. In other words, provided the labeled training (supervised learning) details, the algorithm generates an efficient hyperplane that classifies new instances. SVM is a supervised algorithm for machine learning, which can be used for classification or regression problems. It requires an approach called the kernel trick to rebuild details and then seeks an appropriate boundary between potential outputs based on these transformations. Support vectors are the vectors (cases) that describe the hyperplane. The benefits of SVM are that they can be used to reduce the issues of using linear functions in high-dimensional feature space and the problem of optimization is converted into dual quadratic convex systems. SVM's idea is simple: The algorithm creates a line or hyperplane that divides the data into classes. Multi category SVMs expand the binary SVM to the case of multiple categories and include the binary SVM as a special case. The multi-category SVM enforces the optimum classification rule as the sample size is high, eliminating the sub-optimality of the traditional one-versus-rest strategy. The proposed method deals in a unified manner with the same misclassification costs and the unequal cost case.

\section{METHODOLOGY}

In most of the existing studies, the optimal path is suggested based on various parameters without providing any description of the routes. The proposed method is an automatic system to provide a description of various paths connecting source and destination. Each and every route is described based on mainly three parameters such as traffic congestion, distance, and air quality. The system will also make a suggestion of whether the path is good or bad for traveling. The prime components of the route description system are collecting information of various routes connecting source and destination from Google map API, collecting the congestion details in subroutes using traffic API, collecting distance information using Google distance matrix API, collecting air quality details at subroutes using BreezoMeter API, classification using SVM and route description. The overall system design is illustrated in Fig. 1.

Published By:

Blue Eyes Intelligence Engineering

\& Sciences Publication

(C) Copyright: All rights reserved. 


\section{-}

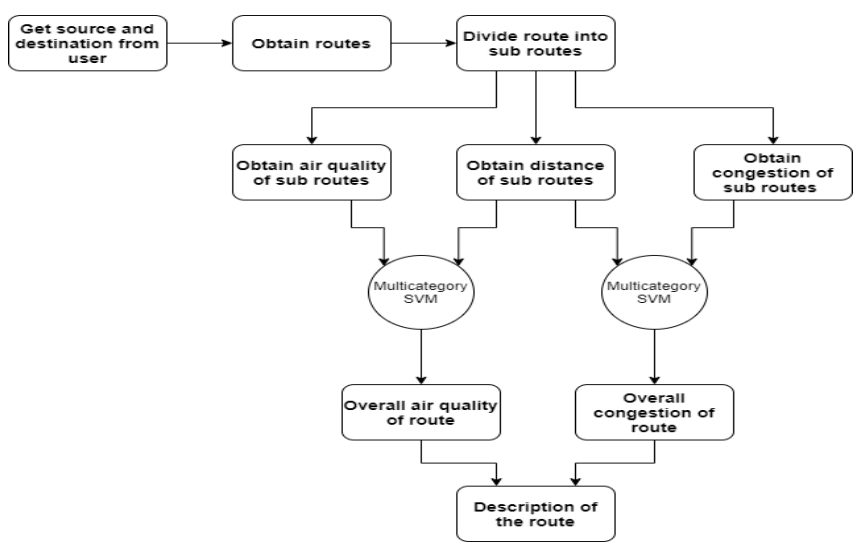

Fig. 1. System design

\section{A. Loading the Map}

With the help of a user interface, users can enter the source and destination. After submitting the required details, the map is displayed using the Google map API. There may be different routes from source to destination. Clicking on the routes will show the distance and time of that particular route. This provides the knowledge seeker with a diagrammatic view of the path. This will also provide the user the service to search what they want in the route such as hotels, ATMs, hospitals, etc and the searched item along the route will be displayed.

\section{B. Determining Route}

After displaying the various routes in the map, the user can enter the waypoint between origin and target. Using the Google map direction API, the longitude and latitude information of subroutes in the particular route from a source location to a destination via a particular waypoint is collected.

\section{Congestion Calculation}

Congestion in traffic is caused by a rise in the number of vehicles on the road or by narrow roads. Since the count of vehicles is high and the road is narrow, the vehicle's individual speed decreases as a consequence of which the time taken to drive increases. With the help of Google Map Traffic API, the proposed method calculates congestion as a reduction in the speed of vehicles running in that particular area.

\section{Distance Calculation}

Distance is also an important factor in finding an optimal route. If the distance is high then this path will not be embraced by people due to the large time required to reach the destination. The distance of different routes between source and destination is obtained from the Google Distance Matrix API. By giving latitude and longitude values of start and endpoint of sub-routes, the API will provide the distance between points.

\section{E. Air Quality Calculation}

Due to various health problems in travelers, air quality is also critical when selecting the routes. Information on air quality is obtained in the proposed system using the Breezometer API which provides data on air quality for a specific region. It accepts the starting and ending points' latitude and longitude details and responds with data in real-time. The details obtained are the air quality levels such as low, poor, moderate, good and excellent. The details of the subroutes are given in the developed system as input to the
Breezometer API and the air quality is obtained in each subroute.

\section{F. Route Classification and Description}

SVM is a simple algorithm that is used in most machine learning works with less computational complexity due to its better accuracy. Although it can be used in both classification and regression issues, SVM is widely used in the field of classification to deliver better results. It works in the task of classification by finding a hyperplane in an $\mathrm{N}$ dimension space that classifies the instances into different classes. Here $\mathrm{N}$ means the number of characteristics each instance uses to classify.

The main objective of multi-class SVM is to assign labels to each participant participating in the process of classification. On the basis of different features, each element will be categorized into different classes using support vector machines and the number of classes will be more than one. This is achieved by reducing the multi-category problem to many problems of binary classification through the one-versus-all approach and is the process of making binary classifiers which makes a distinction between one of the labels and all the others.

Here the multi-class SVM is used for the classification and for a route description. From the Google direction API the intermediate points in the route from source to destination via a particular waypoint are obtained in the form of latitude and longitude. This will make the route to be divided into various segments. For each segment, the three features such as traffic, air quality, and distance are obtained using the above mentioned services. With all of these collected features, air quality and pollution will be graded into different levels with SVM.

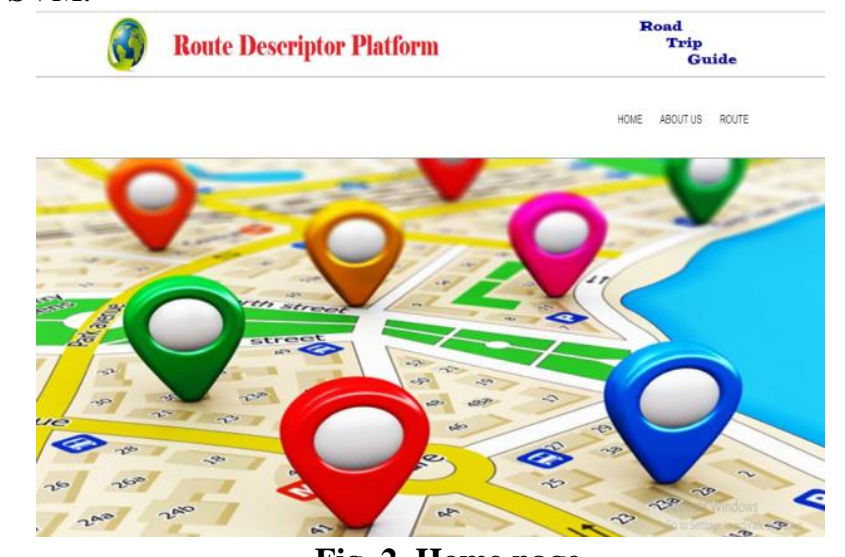

Fig. 2. Home page

\section{G. Algorithm for Descriptive Route}

begin

get the source (s) and destination (d) from user

get the intermediate points between $\mathrm{s}$ and $\mathrm{d}$

for all intermediate points

begin

compute air quality between two adjacent intermediate points (subroute)

compute distance between two adjacent intermediate points (subroute)

compute traffic between two adjacent intermediate points (subroute) end

Published By:

Blue Eyes Intelligence Engineering

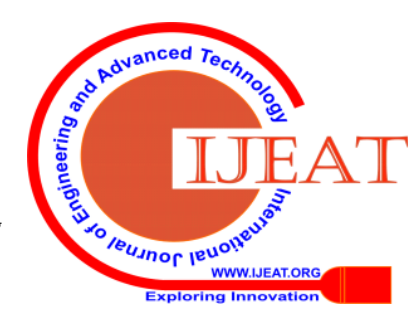


for all subroutes available to multiclass SVM algorithm begin

compute the overall air quality of route from subroute's air quality and distance

compute the overall traffic of route

end

display the description of the route based on obtained air quality and traffic

end

\section{RESULT}

Google will provide a route with less distance and less congestion, but it may not be environmentally friendly.

Some people may have health problems while traveling on a contaminated route. This system provides a detailed overview of the different routes in terms of congestion, distance, air quality and thus provides travelers with the opportunity to decide the route based on their needs. The system functioning as a whole is shown below. The System's "Homepage" is shown in Fig. 2.

After clicking the route section on the homepage, the user will be directed to the route finding page as shown in Fig. 3. The user can enter source and destination and submit in the "Find route" page. Here the user has entered Ernakulam and Kothamangalam as their source and destination.

This will take the consumer into map as shown in Fig. 4. Here are shown the three best routes between Ernakulam and Kothamangalam.

The route definition will be shown after accessing the waypoint between source and destination, as shown in Fig. 5. The description of one of the routes from Ernakulam to Kothamangalam via Muvattupuzha waypoint is given here. Different main points along the path and their distance to the successor point will be shown. The overall air-quality and

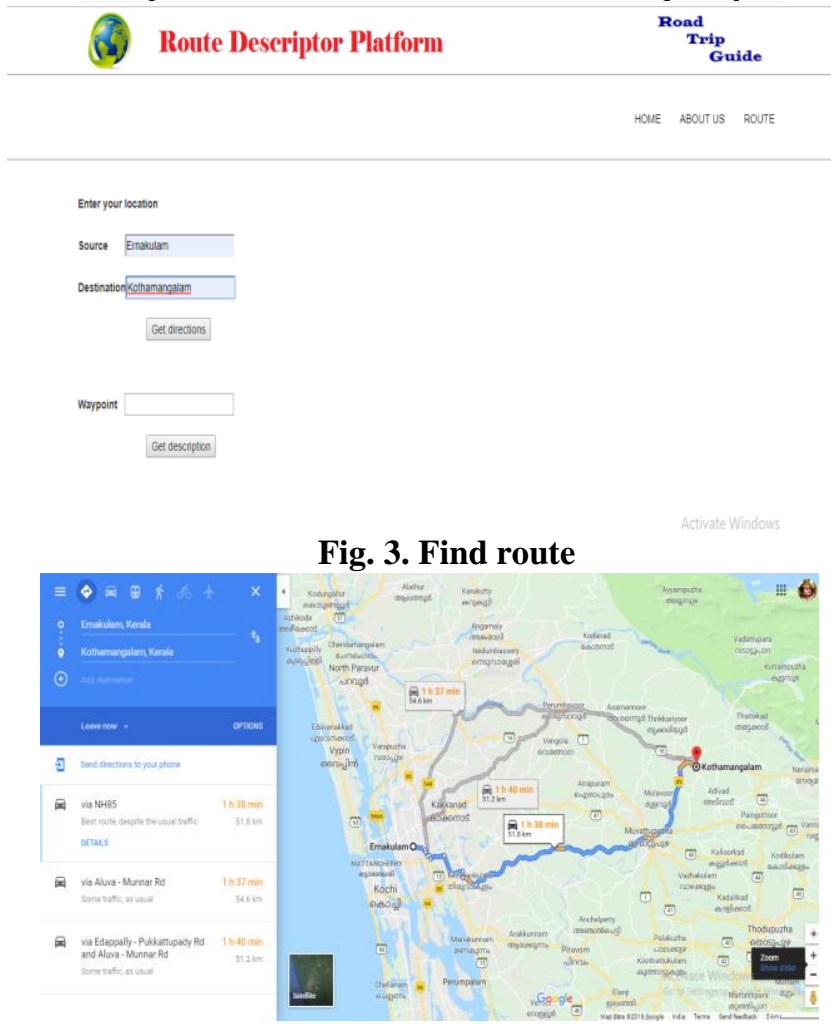

Fig. 4. Map showing route

traffic conditions of the route are outlined along with a brief route summary detailing whether it is appropriate for travel is shown.

\section{PERFORMANCE ANALYSIS}

The emergence of different technologies has changed peoples' lifestyle and many of them choose trips to spend their leisure time. Most people have to travel as part of their work,studies, etc. There are many people who do not know each and every route, and nowadays there is a trend to use the path suggestion systems. The most widely used form of suggestion is Google Maps. The selection of routes is an important task when making recommendations. The quality of existing systems can be enhanced by integrating criteria such as air quality and congestion. The experiments took place nearby Kochi city, one of Kerala's highly polluted and congested areas.

The routes between Kothamangalam and Ernakulam are chosen for performance evaluation. For the route description, the three routes recommended by Google Maps are selected, and the Route Preference Rate (RPR) is calculated using the equation shown in (1).

$$
\mathrm{RPR}=\sum_{i=1}^{N} \frac{\text { distance }_{\mathrm{i}}\left(\mathrm{AQ}_{\mathrm{i}}+\mathrm{TI}_{\mathrm{i}}\right)}{\text { totaldistance }}
$$

where $\mathrm{N}$ is the number of sub-routes, distance $e_{i}$ is the distance of sub route $\mathrm{i}, \mathrm{AQI} \mathrm{I}_{\mathrm{i}}$ is the Air Quality Index of sub route $\mathrm{i}$ and index are 1, 2, 3, 4, and 5 for poor, low, moderate, high, and excellent respectively. $\mathrm{TI}_{\mathrm{i}}$ is the traffic index of sub route $\mathrm{i}$ and index are 1, 2, 3, 4, and 5 for very high, high, moderate, low, and poor respectively whereas, totaldistance is the total distance from the source to destination.

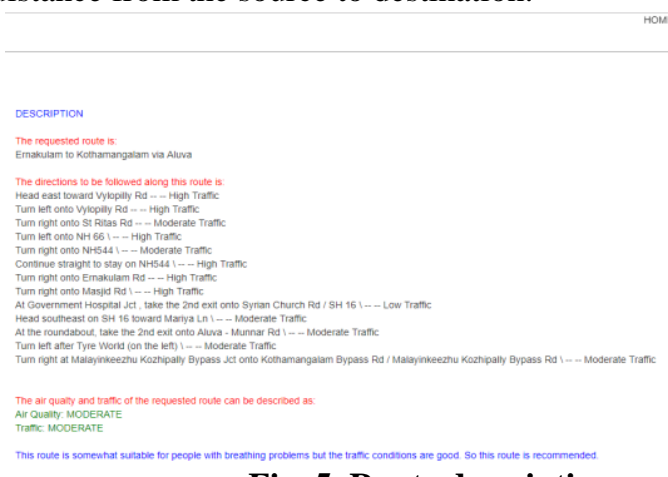

Fig. 5. Route description

Table- I: Route Preference Rate of the Three Routes

\begin{tabular}{|c|c|c|c|}
\hline Source & Destination & Waypoint & Ecofriendliness \\
\hline \multirow{3}{*}{ Ernakulam } & \multirow{3}{*}{ Kothamangalam } & Aluva & 9.53 \\
\cline { 3 - 4 } & & Kakkanad & 13.7 \\
\cline { 3 - 4 } & & Muvattupuzha & 7.92 \\
\hline
\end{tabular}

The ecofriendliness of each route from Ernakulam to Kothamangalam calculated using the above equation is shown in Table 1. Since the measurement of ecofriendliness through the waypoint Kakkanad is higher than the other two, it is the most environmentally friendly road among all.

\section{CONCLUSION}

Traveling through a highly congested and contaminated path is not only a tiring task, but it also creates many health problems for humans.

Published By:

Blue Eyes Intelligence Engineering

\& Sciences Publication

(C) Copyright: All rights reserved.

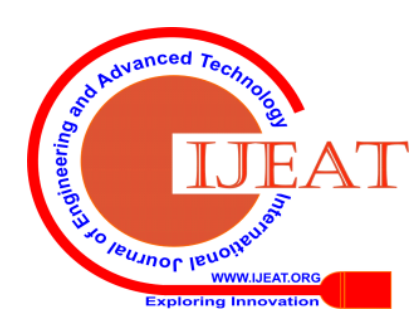


In the current congested and contaminated environment, careful re-routing of vehicles is a necessary task.

Google gives an ideal route in terms of distance and journey time and does not consider air quality. So the recommended path may not be an environmentally friendly road. Paths suggested by Google are the best three. This gives a diagrammatic perspective in the case of traffic and it is very difficult to understand. This system gives a description of different paths from starting point to the destination and the user has the opportunity to find a more environmentally friendly path, taking into account three parameters-air quality, congestion, and distance.

From the results, it could be inferred that the best route varied only marginally from the suggested routes of Google, as the distance choice was higher. But while the preference for congestion parameter and preference for air-quality were weighed more, this percentage of improvement was higher. The recommended route from Google varied greatly when the preference factor for air-quality was considered. The system will also provide a definition of which type of people the route is most suitable for. This method can be expanded by considering some other criteria such as people's reviews to find an optimum path.

\section{REFERENCES}

1. T. Cherrett, B. Waterson, and M. McDonald, "Remote automatic incident detection using inductive loops," in Proceedings of the Institution of Civil Engineers: Transport, 2005, pp. 149-155.

2. G. Palubinskas, F. Kurz, and P. Reinartz, "Traffic congestion parameter estimation in time series of airborne optical remote sensing images," in Proc. of ISPRS Hannover Workshop 2009 - High Resolution Earth Imaging for Geospatial Information,2009.

3. P. S. Maharjan, A. K. Shrestha, "Automatic vehicle detection and road traffic congestion mapping with image processing technique," International Journal of Computer Applications.

4. C. Wenjie, C. Lifeng, C. Zhanglong, and T. Shiliang, "A realtime dynamic traffic control system based on wireless sensor network," in Proceedings of the IEEE ICPPW, Oslo, Norway, 2005, pp. 258-264.

5. S. Roy, S. Bandyopadhyay, M. Das, S. Batabyal, and S. Pal, "Real time traffic congestion detection and management using active RFID and GSM technology," LAP Lambert Academic Publishing, 2012.

6. P. R. Tapar, A. K. Pathrikar, "Automated control system for air pollution detection and rash driving prevention in vehicles," International Journal of Innovative Research in Computer and Communication Engineering, 2017.

\section{AUTHORS PROFILE}

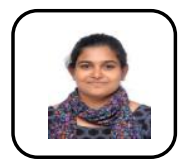

Reshma M earned a Bachelor of Technology in Computer Science and Engineering from Jawaharlal College of Engineering and Technology, Lakkidi in 2018 and currently holds a Master of Technology in Computer Science and Engineering from Mar Athanasius College of Engineering, Kothamangalam, which is affiliated to Abdul Kalam Technical University. Her research interests cover Big Data Analytics, Database Management, and Data Mining.

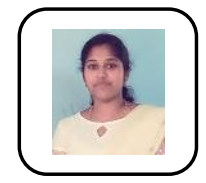

Pristy Paul $\mathbf{T}$ graduated Bachelor of Technology in Computer Science and Engineering from Government Engineering College, Idukki in 2018 and currently holds a Master of Technology in Computer Science and Engineering from Mar Athanasius College of Engineering, Kothamangalam affiliated with APJ Abdul Kalam Technological University. Her research interests include Deep Learning, Artificial Intelligence, and Database Management.

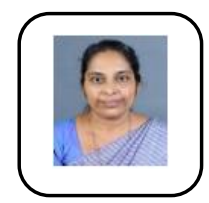

Dr. Surekha Mariam Varghese currently heads the Computer Science and Engineering department, Mar Athanasius College of Engineering, Kothamangalam, Kerala. In 1990, she received her Bachelor of Technology in Computer Science and Engineering from the College of Engineering, Trivandrum affiliated with Kerala University, and a Master in Computer and Information Science Technology from CUSAT, Kochi, in 1996. In 2009, she received a Ph.D. in Computer Security from CUSAT, Kochi. She has accumulated 27 years of teaching and research experience at different Indian institutions. Her research interests cover Machine Learning, Network Security, Database Management, Data Structures and Algorithms, Operating Systems and Distributed Computing.

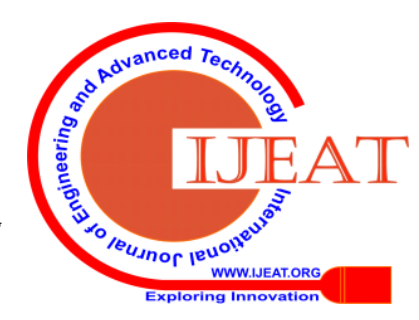

